\title{
Procedimiento de creación de un instrumento virtual para la supervisión administrativa de los ensayos clínicos realizados en el Seguro Social del Perú
}

\section{Procedure of creation of a virtual instrument for the administrative supervision of clinical trials in the Social Security system of Peru}

\section{$\mathrm{DOI}$}

https://doi.org/10.35434/rcmhnaaa.2021.142.1089

Percy Herrera-Añazco ${ }^{1,2, a}$, Suly SotoOrdoñez ${ }^{1, b}$, Maggie Estrada-Martínez ${ }^{1, c}$

Señor editor:

Para que los ensayos clínicos (EC) logre su objetivo de conseguir avances médicos y sirva para identificar estrategias y tratamientos con mejores resultados en beneficios de los pacientes, es necesario el cumplimiento de requisitos éticos y normativos que garanticen la protección a los sujetos de investigación ${ }^{(1)}$. El Instituto de Evaluación de Tecnologías en Salud e Investigación (IETSI), es el órgano descentralizado del Seguro Social (EsSalud) que tiene la función de supervisar, con un enfoque principalmente administrativo, los EC que se desarrollan en los centros de investigación de la institución, de acuerdo con políticas y normas establecidas en el Reglamento de Ensayos Clínicos del Instituto Nacional de salud (INS) y las propias de EsSalud ${ }^{(2)}$.

Del 2015 al 2018, se supervisaron 82 EC ${ }^{(2)}$, siendo en diciembre del 2019, la fecha del último EC evaluado y en espera de supervisión $71 \mathrm{EC}$. Estas supervisiones de carácter presencial, fueron suspendidas como consecuencia de las disposiciones de "distanciamiento social" impuestas por el gobierno central a raíz de la pandemia por la COVID-19 $9^{(3)}$. En ese mismo contexto, el INS, ente rector de la investigación en el país, renovó sus resoluciones y documentos técnicos en el marco de la pandemia COVID-19, integrando los procedimientos virtuales en su normativa, como el RJ N ${ }^{\circ} 139-2020-\mathrm{J}$ OPE/INS y RJ N ${ }^{\circ} 133-2020-J-O P E / I N S^{(4)}$. Debido a ello, la dirección del IETSI dispuso la adaptación de nuestros procesos de tal forma que se reanuden las supervisiones mediante la construcción de un instrumento de carácter virtual.

Tomando como base un formato de evaluación creado por el INS que nos fue proporcionado por la Oficina General de Investigación y Transferencia Tecnológica, el equipo de la Subdirección de Regulación y Gestión de la Investigación en Salud del IETSI procedió a adaptar el formato a uno de modalidad virtual. La matriz de autoevaluación para la supervisión virtual pasó por una prueba piloto en la que se evaluó a dos EC desarrollados en EsSalud, uno en el Centro de Investigación de la Red Asistencial de Lambayeque y el otro en el Centro de Investigación de la Red Prestacional de Rebagliati. Esta prueba piloto nos permitió evaluar la idoneidad del instrumento y la identificación de aspectos perfectible al marco de los lineamientos institucionales y nacionales. Los pasos seguidos en la prueba piloto fueron los siguientes:

Paso 1: Selección de los ensayos clínico a supervisar.

Paso 2: Envió de la solicitud al gerente de la red asistencial con atención al jefe de la Instancia Encargada del Área de Investigación.

Paso 3: Coordinaciones con el investigador principal vía correo electrónico.

Se socializa el instructivo, el link de la matriz de autoevaluación virtual, la hoja complementaria de la ficha de supervisión y la lista de documentos esenciales durante la supervisión.

Se envía el Link de la reunión vía Meet o Zoom.

FILIACIÓN

1. Instituto de Evaluación de Tecnologías en Salud e Investigación - IETSI, EsSalud. Lima, Perú.

2.- Universidad privada san Juan Bautista, Lima, Perú.

a. Médico Nefrólogo.

b. Licenciada en Enfermería.

c. Cirujano Dentista.

ORCID

1. Percy Herrera Añazco

0000-0003-0282-6634

Suly Soto Ordoñe

0000-0003-0284-4605

Maggie Estrada Martínez 0000-0001-5694-7568

\section{CORRESPONDENCIA}

Percy Herrera Añazco

Dirección: Olavegoya 1879 Jesus María. Lima, Perú.

EMAIL

silamud@gmail.com

CONFLICTOS DE INTERÉS

Los autores niegan conflictos de interés.

FINANCIAMIENTO

Autofinanciamiento.

REVISIÓN DE PARES

Recibido: $10 / 06 / 2021$

Aceptado: 30/06/2021

\section{COMO CITAR}

Herrera-Añazco, P., Soto-Ordoñez, S., \& Estrada-Martínez, M. Procedimiento de creación de un instrumento virtual para la supervisión administrativa de los ensayos clínicos realizados en el Seguro Social del Perú. Revista Del Cuerpo Médico Hospital Nacional Revista Del Cuerpo Médico Hospital Nacional 2 Almanzor Aguinaga Asenjo, 2021, 14(2), 244 https://doi.org/10.35434/rcmhnaaa.2021.142 1089

Esta obra está bajo una Licencia Creative Commons Atribución 4.0 Internacional. Versión Impresa: ISSN: 2225-5109 Versión Electrónica: ISSN: 2227-4731 Cross Ref. DOI: 10.35434/rcmhnaaa OJS: https: // cmhnaaa.org.pe/ojs 
Paso 4: Previa a la reunión de supervisión el equipo de supervisión virtual procedió a las siguientes acciones: Revisión y verificación de toda la documentación solicitada.

Reunión de coordinación del equipo de supervisión y creación de la guía final para la entrevista de supervisión virtual que incluyó.

Creación del mecanismo de verificación documentaria mediante un grupo de WhatsApp.

Paso 5: Reunión de supervisión virtual.

Paso 6: Envió del acta final de supervisión virtual.

Las pruebas piloto, permitieron identificar aspectos que nos permitirán mejorar los procesos para que las supervisiones administrativas se desarrollen de la mejor manera, dentro de los cuales podemos señalar:

1.- Las preguntas de la matriz de autoevaluación virtual deben ser claras y precisas y esta debe tener facilidad para subir archivos.

2.- Los archivos de la matriz de autoevaluación virtual deberán archivarse por cada supervisión con resguardo digital de actualización periódica con un sistema de identificación y seguimiento codificado cronológicamente.

3.- La metodología sugerida es entablar diálogo con los evaluados priorizando la indagación de los procesos de la conducción del ensayo clínico, de tal forma que surja, durante el diálogo, los puntos a evaluar según la guía establecida previamente. La guía de la entrevista debe individualizarse de acuerdo a la naturaleza de cada estudio con enfoque de monitoreo basado en riesgos

4.- El equipo de supervisión debe tener una reunión de coordinación previa a la supervisión del estudio, para establecer la selección de preguntas en la guía de entrevista de supervisión según la naturaleza del ensayo clínico; priorizando aspectos como fotos, registros y procesos a consultar.

5.- Las guías de evaluación deben incluir preguntas que nos den una idea del cumplimiento de las Buenas Prácticas Clínicas de los investigadores durante toda la conducción del ensayo clínico.

6. - La entrevista es la fase final de la supervisión virtual, la misma que inicia con el envío del enlace de la matriz de autoevaluación virtual y las indicaciones posteriores de lo que falta incluir en la misma, lo que debería estar completado antes de la reunión de coordinación del equipo de evaluadores donde se diseñará la guía de evaluación.

7.- El equipo evaluador debería estar conformado por tres sub equipos:

Equipo de coordinación administrativa: Este equipo que enviará la matriz de autoevaluación virtual al equipo del centro de investigación seleccionado y verificará que la misma esté completa incluyendo la documentación solicitada. Este equipo mantendrá contacto con el equipo evaluado durante todo el proceso de evaluación virtual hasta la firma del acta. La matriz de autoevaluación virtual deberá estar adecuadamente completa antes de la reunión de coordinación del equipo de evaluadores. Sus miembros serán de una a dos.
Equipo de diseño de evaluación: Este equipo recibirá la información del equipo de coordinación administrativa, estudiará el protocolo del estudio y diseñará las preguntas que se harán en la entrevista virtual en base a la documentación adjuntada y decidirá los documentos adicionales que se solicitarán en el momento de la entrevista. Sus miembros serán de dos a tres.

Equipo de entrevista final: Este equipo dirigirá la entrevista final en base a la guía de supervisión y será el que defina el veredicto final de la entrevista. Sus miembros serán entre dos y tres.

Así mismo, durante la prueba piloto el equipo de la Subdirección de Regulación y Gestión de la Investigación en Salud, identificó algunas ventajas y desventajas de la supervisión virtual, las mismas que se detallan en la tabla 1.

Tabla 1. Ventajas y desventajas de la supervisión virtual de los ensayos clínicos.

\begin{tabular}{ll} 
VENTAJAS & DESVENTAJAS \\
\hline -Optimiza tiempo & -Limitaciones para evaluar las historias \\
-Optimiza procesos & clínicas \\
-Optimiza recursos logísticos & -La entrevista puede sufrir contratiempos \\
-Facilita la descentralización de las & $\begin{array}{l}\text { tanto en video y audio por una señal } \\
\text { inestable de internet. }\end{array}$ \\
supervisiones. & -No se puede realizar una verificación \\
-Mantiene las indicaciones de & exhaustiva de los documentos esenciales y \\
distancia social y trabajo remoto & de los documentos fuente. \\
como medida de bioseguridad & -No se puede verificar en detalle las \\
contra la Covid-19. & instalaciones del centro de investigación. \\
\hline
\end{tabular}

Si bien es cierto esta supervisión virtual no reemplaza la supervisión que los comités institucionales de ética en investigación deben realizar, creemos que esta experiencia puede cumplir con las funciones de supervisiones administrativas que instituciones similares al IETSI deben realizar. De esta manera, se podrá cumplir con la función de verificar el cumplimiento de la normativa que rija el desarrollo de los EC en los países.

\section{REFERENCIAS BIBLIOGRÁFICAS}

1. Martinez R. Clinical Trial Outcomes: What Matters to Patients. JACC Heart Fail. 2019;7(3):272-273. doi: 10.1016/j.jchf.2018.12.003.

2. García-Mostajo JA, Rivera FP, Alvarez-Arroyo V, Catacora-Villasante $M$, Acosta J. Supervisión de ensayos clínicos en hospitales del Seguro Social de Salud del Perú: Enfoque administrativo y regulatorio. Rev Peru Med Exp Salud Publica. 2019;36(4):687-91. doi: 10.17843/rpmesp.2019.364.4569.

3. Herrera-Añazco P, Uyen-Cateriano A, Mezones-Holguin E, TaypeRondan A, Mayta-Tristan P, Malaga G, et al. Some lessons that Peru did not learn before the second wave of COVID-19. Int J Health Plann Manage. 2021;36(3):995-998. doi: 10.1002/hpm.3135.

4. RJ N ${ }^{\circ}$ 139-2020-J-OPE/INS. Pautas para la Ejecución de Ensayos Clínicos durante la Emergencia Sanitaria por Covid-19 [internet]. Perú: Instituto Nacional de Salud 29 de Juno del 2020 [citado el 15 febrero 2021]. Disponible en: https: / / bit.ly/3klOYGj

5. Ministerio de Salud. Acreditación de Comités de Ética. [Internet]. Lima. Registro Peruano de Ensayos Clínicos 2017 [citado el 02 de Julio 2020]. Disponible en: https:// bit.ly/3kkrvFc. 